\title{
INSECTICIDAL ACTIVITY OF SOME MEDICINAL PLANTS OF SRI LANKA
}

\section{C.M. HEWAGE ${ }^{1}$, K.A.N.P. BANDARA ${ }^{2}$, V.KARUNARATNE ${ }^{1}$, B.M.R. BANDARA ${ }^{1}$ and D.S.A. WIJESUNDARA ${ }^{3}$}

'Department of Chemistry, University of Peradeniya, Peradeniya

${ }^{2}$ Central Agricultural Research Institute, Gannoruwa

${ }^{3}$ Royal Botanic Gardens, Peradeniya

(Received: 14 May 1996; accepted: 01 August 1997)

\begin{abstract}
A wide range of solvent extracts prepared from different parts of Sri Lankan medicinal plants (101 extracts from 55 plants) were evaluated for insecticidal activity on the groundnut aphid, Aphis craccivora Koch. and the diamond back moth, Plutella xylostella (L). Of the 94 plant extracts tested for insecticidal activity against Aphis craccivora Koch., Celtis cinnamomea, Cestrum auranticum, Costus specious, Curcuma zedoaria, Hortonia angustifolia, Ocimum gratissimum, Pleiospermium alatum and Zingiber zerumbet reported significantly high mortality (56-90\%). Of the 23 extracts tested for activity against P. xylostella, only Cestrum aurantiacum, Hortonia angustifolia, Pleiospermium alatum, Curcuma zedoaria showed insecticidal activity, low pupation, adult emergence and anti-feedant activity.
\end{abstract}

Key words: Insecticidal activity, medicinal plants.

\section{INTRODUCTION}

Pesticides are an integral aspect of pest control in modern agriculture. However, most synthetic pesticides are associated with a number of undesirable side effects. ${ }^{1}$ Many insects and other plant pathogens have become resistant to chemical pesticides. ${ }^{2}$ For example, 423 species of insects and acarines were resistant by the end of $1980 .^{3}$ Of these 260 species (60\%) are of agricultural importance. Furthermore, due to greater biological productivity in the tropics the development of resistance to insecticides by pests may be more rapid. ${ }^{2}$ Therefore, the discovery of new pest control agents with novel modes of action and greater selectivity on target pests while addressing the human safety concerns remains an urgent need. Many plant species have developed highly sophisticated defence systems, consisting of a complex array of defence chemicals. ${ }^{4}$ These disrupt pest physiology and behavioural activity and provide us with a diverse group of complex chemical structures with different modes of action.

The present study was conducted to identify insecticidal plants of potential value in Sri Lanka. Two agriculturally important insect pests, the groundnut aphid, Aphis craccivora Koch. (Homoptera : Aphididae) and the diamond back moth, Plutella xylostella (L) (Lepidoptera : Yoponomutidae) were used as test 
insects. The groundnut aphid, A. craccivora is a major pest of groundnut and other plants of the Leguminosae. They cause wilting in hot weather but the most serious damage is caused by the transmission of the groundnut rosette virus and other viruses. ${ }^{4}$ The other test insect $P$. xylostella is a very common pest of cabbage and turnip. Severe attacks occur during hot and dry weather.

\section{METHODS AND MATERIALS}

Solvent extracts from 55 plants (94 extracts) were screened against $A$. craccivora (Table 1) while 15 plants (23 extracts) were screened against $P$. xylostella (Table 2).

Plant Material: Plants used in this study were of reproductive maturity. Plant specimens were collected (1-5 kg) from different localities of Sri Lanka, especially from the Central Province and were identified by comparison at the National Herbarium, Royal Botanic Gardens, Peradeniya. The plant material was immediately washed with running water to remove contaminated soil and other surface impurities. They were then cut into small pieces (3-6 cm in length), shade-dried immediately and powdered in a laboratory mill.

Plant Extracts: The air-dried, ground plant material (100 g) was extracted successively with $500 \mathrm{ml}$ each of hot hexane/light petroleum $\left(40-60^{\circ} \mathrm{C}\right)$, dichloromethane, ethyl acetate and methanol in a Soxhlet apparatus. Some were extracted directly with cold ethyl acetate and cold methanol in a bottle shaker. The solubles were concentrated to dryness separately using a rotavapor keeping the water bath temperature below $45^{\circ} \mathrm{C}$. The extracts were subjected to insecticidal activity tests as described below.

Test Insects : A. craccivora was cultured in one-week old cowpea, Vigna ungiculata (L.) Walp., seedlings (cv: Bushitavo) kept in insect proof cages (105 $\mathrm{x} 45 \mathrm{x} 45 \mathrm{~cm}$ ) at a low population level (5 adults per plant). ${ }^{5}$ One-day-old apterous female adults were used in all experiments. They were handled by means of a moistened camel-hair brush.

P. xylostella was cultured on radish plants (cv: Bola Rambu) planted in clay pots ( $15 \mathrm{~cm}$ diameter and $11 \mathrm{~cm}$ high) filled with 1:1:1 mixture of top soil, sand and compost in the laboratory.

All experiments were conducted at a mean temperature of $29.5 \pm 4^{\circ} \mathrm{C}$ and relative humidity (r.h) $80 \pm 4 \%$.A 12 -h photoperiod was maintained by employing a clock mechanism to control four fluorescent tubes (40W) hung over each cage. In all experiments, the moribund insects were counted as dead and mortality counts were taken 24 and $48 \mathrm{~h}$ after introduction (HAI) of insects. The experiments were in completely randomized design (CRD). 
Table 1: Insecticidal activity of some plant extracts (4000 ppm emulsion) against the groundnut aphid, Aphis craccivora in the laboratory.

\begin{tabular}{|c|c|c|c|}
\hline Plant (Family) [Sinhala/Tamil] & Collection & Part ${ }^{n}$ & $\begin{array}{cc}\text { Solvent }^{\mathrm{b}} & \% \\
& \begin{array}{c}\% \\
\text { Mortality }\end{array}\end{array}$ \\
\hline
\end{tabular}

$24 \mathrm{HAT}^{\mathrm{d}}$

+Actinodaphne speciosa Nees.

(Lauraceae) [S-Alikan]

Allaeophania decipens Thw.

(Rubiaceae)

Alpinia abundiflora Burt \& Smith

(Zingiberaceae)

+ Alpinia fax Burtt \& Smith

(Zingiberaceae)

Alpinia nigra (Gaertn.) Burtt

(Zingiberaceae) [S-Alugas, T-Shittai rattai]

Anotis richardiana (Arn.) Hook. f.

(Rubiaceae)

Artimisia dubia var. grata Wall

(Compositae) [S-Walkolondu, T-Marukolondu]

Bridelia retusa (L.) Spreng

(Euphorbiaceae) [S-Ketakala, T-Adamarudu]

Butea monosperma (Lam.) Taub.

(Leguminosae) [S-Gaslela, T-Parasu]

Celtis cinnamomea Lindl. ex Planch.

(Ulmaceae) [S-Gurenda, T-Pinari]

Cestrum auranticum Lindl.

(Solanaceae)

Chrysopogon zeylanicus (steud.) Thw.

(Gramaceae) [S-Gawara]

Costus specious (Koen.) Sm

(Zingiberaceae) [S-Tebu, T-Khoshadam]

Curcuma longa $\mathrm{L}$.

(Zingiberaceae) [S-Kaha, T-Manchal]

Curcuma zeadoria (Berg.) Roscoe.

(Zingiberaceae) [S-Harankaha, T-Kasturimangal]

Eupatorium inulifolium HBK.

(Compositae)

Eupatorium odoratum L.

(Compositae) [S-Podisinghomaran]

Eupatorium riparium Regel.

(Compositae)

Gaultheria rudis Stapt.

(Ericaceae) [S-Wal kapuru]

Glochidion montanum Thw.

(Euphorbiaceae)

Gynostemma laxum (Wall.) Cong.

(Curcubitaceae)

\begin{tabular}{|c|c|c|c|}
\hline Horton Plains & Lf & $\mathrm{MeOH}^{\mathrm{h}}$ & $10.00 \mathrm{~d}$ \\
\hline \multirow[t]{2}{*}{ Horton Plains } & $\mathrm{Ap}$ & $\mathrm{MeOH}^{\mathrm{h}}$ & $13.33 \mathrm{~d}$ \\
\hline & St & $\mathrm{MeOH}^{\mathrm{h}}$ & \\
\hline \multirow[t]{3}{*}{ Horton Plains } & $\mathrm{Rh}$ & ${ }^{*}$ Pet" & $13.33 d$ \\
\hline & & ${ }^{*} \mathrm{CH}_{2} \mathrm{Cl}_{2}{ }^{11}$ & $6.66 \mathrm{~d}$ \\
\hline & $\mathrm{Th}$ & ${ }^{*} \mathrm{MeOH}^{\mathrm{h}}$ & $\begin{array}{c}13.33 \mathrm{~d} \\
0.0 \mathrm{~d}\end{array}$ \\
\hline Rakwana & Ib. & $\mathrm{MeOH}^{\prime \prime}$ & $0.0 \mathrm{~d}$ \\
\hline \multirow[t]{2}{*}{ Hakgala } & $\mathrm{Ft}$ & $\mathrm{MeOH}^{\mathrm{n}}$ & $20.0 \mathrm{~d}$ \\
\hline & $\mathrm{Tb}$ & $\mathrm{MeOH}^{\prime \prime}$ & $13.33 \mathrm{~d}$ \\
\hline Pattipola & $\mathrm{Wp}$ & $\mathrm{MeOH}^{\mathrm{l}}$ & $6.66 \mathrm{~d}$ \\
\hline \multirow[t]{6}{*}{ Ambawela } & $\mathrm{Lf}$ & $\mathrm{MeOH}^{\mathrm{c}}$ & $3.33 \mathrm{~d}$ \\
\hline & Lf & $\mathrm{MeOH}^{\mathrm{h}}$ & $0.00 \mathrm{~d}$ \\
\hline & St & $\mathrm{MeOH}^{\mathrm{c}}$ & $6.66 \mathrm{~d}$ \\
\hline & St & $\mathrm{MeOH}^{h}$ & $3.33 \mathrm{~d}$ \\
\hline & Rt & $\mathrm{MeOH}^{\mathrm{c}}$ & $3.33 \mathrm{~d}$ \\
\hline & Rt & $\mathrm{MeOH}^{\mathrm{h}}$ & $0.00 \mathrm{~d}$ \\
\hline Hakgala & Lf & $\mathrm{MeOH}^{\mathrm{h}}$ & $13.33 \mathrm{~d}$ \\
\hline Mahiyangana & St Bk & $\mathrm{EtOH}^{\mathrm{c}}$ & $9.99 \mathrm{~d}$ \\
\hline \multirow[t]{2}{*}{ Hakgala } & Lf & $\mathrm{MeOH}^{\mathrm{h}}$ & $83.33 \mathrm{a}$ \\
\hline & St & $\mathrm{MeOH}^{\mathrm{h}}$ & \\
\hline \multirow{2}{*}{ Hakgala } & Lf & $\mathrm{MeOH}^{n}$ & $56.66 \mathrm{c}$ \\
\hline & St & $\mathrm{MeOH}^{\mathrm{h}}$ & \\
\hline Hakgala & Wp & $\mathrm{MeOH}^{\mathrm{n}}$ & $13.33 \mathrm{~d}$ \\
\hline Kalutara & $\mathrm{Rh}$ & $\mathrm{MeOH}^{\mathrm{n}}$ & $90.00 \mathrm{a}$ \\
\hline \multirow[t]{2}{*}{ Kandy } & $\mathrm{Tb}$ & $\mathrm{EtOH}^{\mathrm{c}}$ & $10.00 \mathrm{~d}$ \\
\hline & $\mathrm{Tb}$ & $\mathrm{MeOH}^{\mathrm{c}}$ & $3.33 \mathrm{~d}$ \\
\hline Kandy & $\mathrm{Tb}$ & $\mathrm{MeOH}^{\mathrm{h}}$ & $73.33 \mathrm{bc}$ \\
\hline \multirow[t]{2}{*}{ Hakgala } & St & $\mathrm{MeOH}^{\mathrm{h}}$ & $0.00 \mathrm{~d}$ \\
\hline & St & $\mathrm{MeOH}^{\mathrm{h}}$ & $3.33 \mathrm{~d}$ \\
\hline Kandy & Lf & $\mathrm{MeOH}^{\mathrm{h}}$ & $6.66 \mathrm{~d}$ \\
\hline \multirow[t]{3}{*}{ Hakgala } & Ap & $\mathrm{MeOH}^{\mathrm{h}}$ & $0.00 \mathrm{~d}$ \\
\hline & Rt & ${ }^{*} \mathrm{Hex}^{\mathrm{h}}$ & $3.33 \mathrm{~d}$ \\
\hline & & ${ }^{*} \mathrm{CH}_{2} \mathrm{Cl}_{2}{ }^{\mathrm{b}}$ & $13.33 \mathrm{~d}$ \\
\hline \multirow[t]{2}{*}{ Horton Plains } & Wp-Lf & $\mathrm{MeOH}^{2}{ }^{2}$ & $6.66 \mathrm{~d}$ \\
\hline & Lf & $\mathrm{MeOH}^{\mathrm{h}}$ & $0.00 \mathrm{~d}$ \\
\hline Hakgala & Lf & $\mathrm{MeOH}^{\mathrm{n}}$ & $6.66 \mathrm{~d}$ \\
\hline Hakgala & $\mathrm{Ap}$ & $\mathrm{MeOH}^{\mathrm{h}}$ & $9.99 \mathrm{~d}$ \\
\hline \multirow[t]{2}{*}{ Sinharaja } & Lf & $\mathrm{MeOH}^{h}$ & $73.33 \mathrm{bc}$ \\
\hline & St & $\mathrm{MeOH}^{\mathrm{h}}$ & $3.33 \mathrm{~d}$ \\
\hline
\end{tabular}


Table 1 Contd.

Plant (Family) [Sinhala/Tamil]

$\begin{array}{lccc}\text { Collection } & \text { Part }^{\mathrm{a}} & \text { Solvent }^{\mathrm{b}} & \begin{array}{c}\% \\ \text { Mortality }^{\mathrm{C}}\end{array}\end{array}$

24 HAT $^{d}$

+Hortonia angustifolia (Thw.) Trim.

(Monimiaceae)

+Hortonia floribunda Wight ex Arn.

(Monimiaceae) [S-Gawara]

Hypericum mysurense Wight \& Arn.

(Gutiferae)

Justicia betonia L.

(Acanthaceae) [S-Sudu puruk]

Leucas biflora (Vahl.) Benth.

(Labiatae) [S-Gata Tumba, T-Peyt tumpi]

Leucas zeylanica (L.) Benth.

(Labiatae) [S-Geta Tumba, T-Mudi tumpi]

Lobelia aromatica Moon ex Wight

(Lobeliaceae)

Lobelia nicotianifolia Roth ex R. \& Schult

(Lobeliaceae) [S-Waldunkola, T-Kattupoillai]

+Mastixia tetranda (Wight ex Thwaites) C.B.Clarke Hakgala

(Cornaceae) [S-Matawara]

Ocimum gratissimum $\mathrm{L}$.

(Labiatae) [S-Gastala, T-Elumichantulasi]

Osbeckia cupularis D. Don ex Wight \& Arn.

(Melastomaceae) [S-Bowitia]

Pedalium murex L.

(Pedaliaceae) [S-Ethnerenchi, T-Perunerenchi]

Phyllanthus embilica L.

(Euphorbiaceae) [S-Ambula, T-Nelli]

Phyllanthus reticulatus Poir.

(Euphorbiaceae) [S-Welkayila, T-Pulla]

Pimenta officinalis Lindl.

(Myrtaceae)

Pleiospermium alatum (Wight \& Arn.) Swingle Sigiriya

(Rutaceae) [S-Tumpathkurundu, T-Mailadidurundu]

\begin{tabular}{|c|c|c|c|}
\hline Hakgala & Rt & $\mathrm{MeOH}^{\mathrm{h}}$ & $6.66 \mathrm{~d}$ \\
\hline \multirow[t]{3}{*}{ Hakgala } & Lf & $\mathrm{MeOH}^{\mathrm{h}}$ & $10.00 \mathrm{~d}$ \\
\hline & St & $\mathrm{MeOH}^{\mathrm{h}}$ & $6.66 \mathrm{~d}$ \\
\hline & Rt & $\mathrm{MeOH}^{\mathrm{h}}$ & $3.33 \mathrm{~d}$ \\
\hline \multirow[t]{2}{*}{ Pattipola } & Lf & $\mathrm{MeOH}^{\mathrm{h}}$ & $6.66 \mathrm{~d}$ \\
\hline & St & $\mathrm{MeOH}^{\mathrm{h}}$ & $13.33 \mathrm{~d}$ \\
\hline Kandy & Ap & $\mathrm{MeOH}^{\mathrm{h}}$ & $3.33 \mathrm{~d}$ \\
\hline Horton Plains & $W p$ & $\mathrm{MeOH}^{\mathrm{h}}$. & $10.00 \mathrm{~d}$ \\
\hline Hakgala & Wp & $\mathrm{MeOH}^{\mathrm{h}}$ & $3.33 \mathrm{~d}$ \\
\hline \multirow[t]{2}{*}{ Horton Plains } & Lf & $\mathrm{MeOH}^{\mathrm{h}}$ & $10.00 \mathrm{~d}$ \\
\hline & St Bk & $\mathrm{MeOH}^{\mathrm{h}}$ & $3.33 \mathrm{~d}$ \\
\hline \multirow[t]{2}{*}{ Hakgala } & Lf & $\mathrm{MeOH}^{\mathrm{h}}$ & $6.66 \mathrm{~d}$ \\
\hline & St Bk & $\mathrm{MeOH}^{\mathrm{h}}$ & $10.00 \mathrm{~d}$ \\
\hline Hakgala & Lf & $\mathrm{MeOH}^{\mathrm{h}}$ & $13.33 \mathrm{~d}$ \\
\hline \multirow[t]{2}{*}{ Kandy } & Ap & $\mathrm{MeOH}^{\mathrm{h}}$ & $73.33 \mathrm{bc}$ \\
\hline & $\mathrm{Rt}$ & $\mathrm{MeOH}^{\mathrm{h}}$ & $86.66 \mathrm{ab}$ \\
\hline Horton Plains & Wp & $\mathrm{MeOH}^{\mathrm{h}}$ & $10.00 \mathrm{~d}$ \\
\hline Puttalum & Lf & $\mathrm{MeOH}^{\mathrm{h}}$ & $3.33 \mathrm{~d}$ \\
\hline Hakgala & Lf & $\mathrm{MeOH}^{\mathrm{h}}$ & $6.66 \mathrm{~d}$ \\
\hline Sigiriya & $\mathrm{Lf}$ & $\mathrm{MeOH}^{\mathrm{h}}$ & $13.33 \mathrm{~d}$ \\
\hline \multirow[t]{2}{*}{ Kandy } & Lf & $\mathrm{MeOH}^{\mathrm{h}}$ & $3.33 \mathrm{~d}$ \\
\hline & St Bk & $\mathrm{MeOH}^{\mathrm{h}}$ & $6.66 \mathrm{~d}$ \\
\hline \multirow{4}{*}{ Sigiriya } & St Bk & ${ }^{*} \mathrm{Hex}{ }^{\mathrm{h}}$ & $90.00 \mathrm{a}$ \\
\hline & Rt Bk & ${ }^{*} \mathrm{EtOAc}^{\mathrm{h}}$ & $3.33 \mathrm{~d}$ \\
\hline & & *EtOAc ${ }^{\mathrm{h}}$ & $6.66 \mathrm{~d}$ \\
\hline & & $* \mathrm{MeOH}^{\mathrm{h}}$ & $0.00 \mathrm{~d}$ \\
\hline \multirow[t]{2}{*}{ Horton Plains } & Lf & $\mathrm{MeOH}^{\mathrm{h}}$ & $13.33 \mathrm{~d}$ \\
\hline & St & $\mathrm{MeOH}^{\mathrm{h}}$ & $3.33 \mathrm{~d}$ \\
\hline \multirow[t]{2}{*}{ Horton Plains } & Lf & $\mathrm{MeOH}^{\mathrm{h}}$ & $10.00 \mathrm{~d}$ \\
\hline & St & $\mathrm{MeOH}^{\mathrm{h}}$ & $6.66 \mathrm{~d}$ \\
\hline Sigiriya & St Bk & $\mathrm{MeOH}^{\mathrm{h}}$ & $3.33 \mathrm{~d}$ \\
\hline \multirow[t]{2}{*}{ Kandy } & Lf & $\mathrm{MeOH}^{\mathrm{h}}$ & $13.33 \mathrm{~d}$ \\
\hline & St Bk & $\mathrm{MeOH}^{\mathrm{h}}$ & $6.66 \mathrm{~d}$ \\
\hline \multirow[t]{2}{*}{ Hakgala } & $\mathrm{Lf}$ & $\mathrm{MeOH}^{\mathrm{h}}$ & $10.00 \mathrm{~d}$ \\
\hline & St & $\mathrm{MeOH}^{\mathrm{h}}$ & $0.00 \mathrm{~d}$ \\
\hline \multirow[t]{3}{*}{ Hakgala } & Lf & $\mathrm{MeOH}^{\mathrm{h}}$ & $6.66 \mathrm{~d}$ \\
\hline & St & $\mathrm{MeOH}^{\mathrm{h}}$ & $13.33 \mathrm{~d}$ \\
\hline & St Bk & $\mathrm{MeOH}^{\mathrm{h}}$ & $0.00 \mathrm{~d}$ \\
\hline
\end{tabular}

Psychotria bisculata (Wight \& Arn.)

(Rubiaceae)

Psychotria nigra van cronata Hk.f

(Rubiaceae)

Ricinus communis $\mathrm{L}$.

(Euphorbiaceae) [S-Erandu, T-Chittamanakku]

Saprosma foetens (Wight) K. schum.

(Rubiaceae)

Sarcococca pruniformis Lindl.

(Buxaceae)

+Semecarpus coriacea Thw.

(Anarcardiaceae) [S-Badulla] 
Table 1 Contd.

\begin{tabular}{|c|c|c|c|c|}
\hline Plant (Family) [Sinhala/Tamil] & Collection & Part $^{\mathrm{a}}$ & Solvent ${ }^{b}$ & $\begin{array}{c}\% \\
\text { Mortality }^{c} \\
24 \text { HAT }^{d}\end{array}$ \\
\hline $\begin{array}{l}+ \text { Semicarpus nigro-viridis Thw. } \\
\text { (Anarcardiaceae) [S-Getabadulla] }\end{array}$ & Hakgala & $\begin{array}{l}\text { Lf } \\
\text { St } \\
\text { St Bk }\end{array}$ & $\begin{array}{l}\mathrm{MeOH}^{\mathrm{h}} \\
\mathrm{MeOH}^{\mathrm{h}} \\
\mathrm{MeOH}^{\mathrm{h}}\end{array}$ & $\begin{array}{r}10.00 \mathrm{~d} \\
6.66 \mathrm{~d} \\
13.33 \mathrm{~d}\end{array}$ \\
\hline $\begin{array}{l}\text { +Semicarpus obscura Thw. } \\
\text { (Anarcardiaceae) [S-Badulla] }\end{array}$ & Hakgala & $\begin{array}{l}\text { Lf } \\
\text { St } \\
\text { St Bk }\end{array}$ & $\begin{array}{l}\mathrm{MeOH}^{\mathrm{h}} \\
\mathrm{MeOH}^{\mathrm{h}} \\
\mathrm{MeOH}^{\mathrm{h}}\end{array}$ & $\begin{array}{r}13.33 \mathrm{~d} \\
3.33 \mathrm{~d} \\
6.66 \mathrm{~d}\end{array}$ \\
\hline $\begin{array}{l}\text { Sesamum indicum L. } \\
\text { (Pedaliaceae) [S-Walthala, T-Ellu] }\end{array}$ & Rambukkana & Lf & $\mathrm{EtOH}^{\mathrm{c}}$ & $10.00 \mathrm{~d}$ \\
\hline $\begin{array}{l}\text { Solanum giganteum Jacq. } \\
\text { (Solanaceae) }\end{array}$ & Hakgala & $\begin{array}{l}\text { Ft } \\
\text { Ft }\end{array}$ & $\begin{array}{l}\mathrm{MeOH}^{\mathrm{c}} \\
\mathrm{MeOH}^{\mathrm{h}}\end{array}$ & $\begin{array}{l}9.99 \mathrm{~d} \\
3.33 \mathrm{~d}\end{array}$ \\
\hline $\begin{array}{l}\text { Strobilanthes auriculatus Nees. } \\
\text { (Acanthaceae) }\end{array}$ & Horton Plains & $\begin{array}{l}\text { Lf } \\
\text { St } \\
\text { Rt }\end{array}$ & $\begin{array}{l}\mathrm{MeOH}^{\mathrm{h}} \\
\mathrm{MeOH}^{\mathrm{h}} \\
\mathrm{MeOH}^{\mathrm{h}}\end{array}$ & $\begin{array}{r}10.00 \mathrm{~d} \\
3.33 \mathrm{~d} \\
6.66 \mathrm{~d}\end{array}$ \\
\hline $\begin{array}{l}\text { Strobilanthes hookeri Nees. } \\
\text { (Acanthaceae) }\end{array}$ & Horton Plains & $\begin{array}{l}\text { Lf } \\
\text { St }\end{array}$ & $\begin{array}{l}\mathrm{MeOH}^{\mathrm{h}} \\
\mathrm{MeOH}^{\mathrm{h}}\end{array}$ & $\begin{array}{r}13.33 \mathrm{~d} \\
3.33 \mathrm{~d}\end{array}$ \\
\hline Strobilanthes viscosa (Nees) T. Anders. & Horton Plains & $\begin{array}{l}\text { Lf } \\
\text { St }\end{array}$ & $\begin{array}{l}\mathrm{MeOH}^{\mathrm{h}} \\
\mathrm{MeOH}^{\mathrm{h}}\end{array}$ & $\begin{array}{l}9.99 \mathrm{~d} \\
0.00 \mathrm{~d}\end{array}$ \\
\hline $\begin{array}{l}\text { Theobroma cacao L. } \\
\text { (Sterculaceae) [S-Cocoa, T-Coco] }\end{array}$ & Kalutara & $\mathrm{Ft}$ & EtOAc $c^{c}$ & $10.00 \mathrm{~d}$ \\
\hline $\begin{array}{l}\text { Thespesia populnea (L.) Soland ex Corr. } \\
\text { (Malvaceae) [S-Gansuriya, T-Kavarachu] }\end{array}$ & Kandy & $\begin{array}{l}\text { Lf } \\
\text { St }\end{array}$ & $\begin{array}{l}\mathrm{MeOH}^{\mathrm{h}} \\
\mathrm{MeOH}^{\mathrm{h}}\end{array}$ & $\begin{array}{l}6.66 \mathrm{~d} \\
3.33 \mathrm{~d}\end{array}$ \\
\hline $\begin{array}{l}\text { Ulex europaeus } \mathrm{L} \text {. } \\
\text { (Leguminosae) }\end{array}$ & Horton Plains & $\mathrm{Wp}$ & $\mathrm{MeOH}^{\mathrm{h}}$ & $0.00 \mathrm{~d}$ \\
\hline $\begin{array}{l}\text { Wendlandia bicuspidata Wight \& Arn. } \\
\text { (Rubiaceae) [S-Balalhulla] }\end{array}$ & Hakgala & $\begin{array}{l}\text { Lf } \\
\text { St } \\
\text { St Bk }\end{array}$ & $\begin{array}{l}\mathrm{MeOH}^{\mathrm{h}} \\
\mathrm{MeOH}^{\mathrm{h}} \\
\mathrm{MeOH}^{\mathrm{h}}\end{array}$ & $\begin{array}{r}10.00 \mathrm{~d} \\
0.00 \mathrm{~d} \\
9.99 \mathrm{~d}\end{array}$ \\
\hline $\begin{array}{l}\text { Zingiber zerumbert (L.) Sm. } \\
\text { (Zingiberaceae) [S-Walinguru] } \\
\text { Untreated Control } \\
\text { CV (Coefficient of Variation) }\end{array}$ & Hakgala & $\mathrm{Tb}$ & $\mathrm{MeOH}^{\mathrm{h}}$ & $\begin{array}{l}60.00 \mathrm{~d} \\
13.33 \mathrm{~d} \\
13.58\end{array}$ \\
\hline
\end{tabular}

$+:$ Species endemic to Sri Lanka

$\because$ : Successive extraction

$a$ : Ap - Aerial Part; Lf - Leaf; Rh - Rhizome; Rt - Root; St Bk - Stem Bark; Tb - Tuber

$b: \mathrm{h}$ - Hot extraction; Hex - $\underline{\mathrm{n}}$-hexane

$c:$ Mean of 3 replicates each containing 10 aphids

$d$ : Hours after treatment

Means followed by the same letter are not significantly different $(p \geq 0.05)$ according to Duncan's Multiple Range Test. Arc sign percentage transformation was used.

Relative toxicity against $A$. craccivora ${ }^{5,6}$ : The spray solutions were prepared as follows: each extract ( $64 \mathrm{mg}$ ) was mixed with $128 \mathrm{mg}$ of the detergent, LF $12 \mathrm{R}$ (supplied by Ciba-Geigy Ltd., Basel, Switzerland), in 1:2 (w/w) ratio and was dissolved in analytical grade methanol or acetone and diluted with distilled water in $1: 9(\mathrm{v} / \mathrm{v})$ ratio to obtain $16 \mathrm{ml}$ of a $4000 \mathrm{ppm}$ emulsion. 
Fifteen aphids were placed on the underside of a detached young (one-weekold) cowpea leaf. Each leaf was placed on a moistened filter paper (Whatman No. 1) kept in the basal plate of a petri dish (9 $\mathrm{cm}$ diameter). After the aphids had settled (about $30 \mathrm{~min}$ ), each leaf was turned upside down and sprayed with a $4000 \mathrm{ppm}$ emulsion of an extract using a Potter's spray tower. For each spraying, $4.0 \mathrm{ml}$ of the above emulsion was used in the spray tower under a pressure of $20 \mathrm{~g} \mathrm{~cm}^{-2}$. The leaf was turned right-side up and the base of the petiole was inserted into a moistened cotton plug to slow down desiccation. The basal plate of each petri dish was covered with a tight-fitting plastic lid containing windows of fine gauze. A mixture of the particular solvent, distilled water and the detergent was used as standard control and distilled water alone as an untreated control. The experiment was replicated four times. Mortality counts were taken 24 and $48 \mathrm{HAI}$.

Table 2: Insecticidal activity of some plant extracts against the diamond back moth, Plutella xylostella in the laboratory (Only the plants which showed significant activity are listed).

\begin{tabular}{|c|c|c|c|c|c|}
\hline $\begin{array}{l}\text { Plant Extract }\left(\text { Part }^{\mathrm{a}} \text {, }\right. \\
\left.\text { Solvent } \mathrm{t}^{\mathrm{b}}\right)\end{array}$ & $\begin{array}{l}\text { \% Mortality } \\
24 \text { HAT }^{\mathrm{d}}\end{array}$ & $48 \mathrm{HAT}$ & $\begin{array}{c}\% \\
\text { Pupation }\end{array}$ & $\begin{array}{l}\% \text { Adult } \\
\text { Emergence }\end{array}$ & $\begin{array}{c}\text { Feeding Area } \\
\mathrm{mm}^{2} \\
48 \mathrm{HAI}^{\mathrm{e}}\end{array}$ \\
\hline $\begin{array}{l}\text { Hortonia angustifolia } \\
\text { (Thw.) Trim. } \\
\text { (Rt, } \mathrm{MeOH}^{\mathrm{n}} \text { ) }\end{array}$ & $86.66 \mathrm{a}$ & $86.66 \mathrm{a}$ & $0.00 \mathrm{c}$ & $0.00 \mathrm{~b}$ & $16.60 \mathrm{de}$ \\
\hline $\begin{array}{l}\text { Pleiospermium alatum } \\
\text { (Wight \& Arn.) Swingle } \\
\text { (St Bk, Hex") }\end{array}$ & $93.33-\mathrm{a}$ & $93.33 \mathrm{a}$ & $0.00 c$ & $0.00 \mathrm{c}$ & $38.00 \mathrm{~d}$ \\
\hline $\begin{array}{l}\text { Cestrum auranticum Lindl. } \\
\left(\mathrm{Lf}, \mathrm{MeOH}^{\mathrm{h}} \text { ) }\right.\end{array}$ & $23.33 \mathrm{bc}$ & $80.00 \mathrm{ab}$ & $6.66 \mathrm{~b}$ & $3.33 \mathrm{~b}$ & $18.60 \mathrm{de}$ \\
\hline $\begin{array}{l}\text { Costus specious (Koen.) } \\
\mathrm{Sm} \\
\left(\mathrm{Rh}, \mathrm{MeOH}^{\mathrm{h}}\right)\end{array}$ & $13.33 \mathrm{~cd}$ & $40.00 \mathrm{bc}$ & $10.0 \mathrm{~b}$ & $3.33 \mathrm{~b}$ & $73.30 \mathrm{c}$ \\
\hline $\begin{array}{l}\text { Curcuma zedoaria (Berg.) } \\
\text { Roscoe. } \\
\left(\mathrm{Tb}, \mathrm{MeOH}^{\mathrm{h}} \text { ) }\right.\end{array}$ & $40.00 \mathrm{~b}$ & $53.33 \mathrm{bc}$ & $0.00 \mathrm{c}$ & $0.00 \mathrm{~b}$ & $0.00 \mathrm{e}$ \\
\hline $\begin{array}{l}\text { Zingiber zerumbet (L.) Sm } \\
\left(\mathrm{Tb}, \mathrm{MeOH}^{\mathrm{h}}\right)\end{array}$ & $40.00 \mathrm{~b}$ & $53.33 \mathrm{bc}$ & $10.0 \mathrm{~b}$ & $0.00 \mathrm{~b}$ & $96.60 \mathrm{~b}$ \\
\hline Untreated Control & $3.33 \mathrm{~d}$ & $16.66 \mathrm{c}$ & $70.0 \mathrm{a}$ & $66.66 \mathrm{a}$ & $198.60 \mathrm{a}$ \\
\hline $\mathrm{CV}$ & 24.04 & 28.93 & 46.40 & 60.82 & 19.88 \\
\hline
\end{tabular}

$a$ : Lf - Leaf; Rh - Rhizome; Rt - Root; St Bk - Stem Bark; Tb - Tuber

$b:$ Hot extraction

$c:$ Mean of the three replicates each containing ten larvae

$d$ : Hours after treatment

$e:$ Hours after introduction of larvae

In each column, the letters followed by the same letter are not significantly different $(\mathrm{p} \leq 0.05)$ according to DMRT. Arc sign percentage transformation was used. 
Relative toxicity against $P$. xylostella: Cabbage leaf disks (9 $\mathrm{cm}$ diameter) were dipped in $4000 \mathrm{ppm}$ emulsion of each extract and were left to air dry. The dry disks were offered to 10 , second instar larvae of $P$. xylostella obtained from the above culture. The cabbage leaf disks and larvae were confined into a glass petri $\operatorname{dish}(9 \mathrm{~cm}$ diameter) with a moistened filter paper placed at the bottom. Cabbage leaf disk dipped in the solution without the plant extract was used as the untreated control. The treatments were replicated three times and arranged in $\mathrm{CRD}$ in the laboratory. After the introduction, larval mortality and their developmental abnormalities, if any, were recorded daily. The leaf area consumed by the 10 larvae were recorded at $48 \mathrm{HAI}$ using a graph paper, and then the treated leaf disks were replaced by fresh untreated ones.

Evaluation of the field performance of Hortonia angustifolia: Field performance of Hortonia angustifolia (Rt) methanol extract against cabbage leaf eating caterpillars was comparatively evaluated, with neem oil (Kandy Ayurvedic Drugs Ltd.) and some other commercially available pesticides, at the open prison camp Pallekalle, in the Kandy district (Table 3). In this experiment, cabbage (cv : SD cross) was planted in plots of $12 \mathrm{~m}^{2}$ at a spacing of $40 \times 50 \mathrm{~cm}$; each plot was separated by a one-meter wide strip of land. Different treatments were sprayed to individual plots, using a high volume knapsack sprayer, at 10 day intervals commencing two weeks after transplanting. Each treatment of insecticide was replicated three times and arranged in Randomized Complete Block Design (RCBD).

Table 3: The comparative efficacy of different pest-control agents against-leaf eating caterpillars of cabbage - Yala 1989.

\begin{tabular}{lcccc}
\hline Insecticide & $\begin{array}{c}\text { Rate (g or } \\
\text { 1/ha) }\end{array}$ & No. of larvae & $\begin{array}{c}\text { Leaf } \\
\text { damage }\end{array}$ & $\begin{array}{c}\% \text { yield } \\
\text { loss }\end{array}$ \\
\hline $\begin{array}{l}\text { Triflumuron } \\
\text { (Alsystine 480 SC) }\end{array}$ & $200-400 \mathrm{~g}$ (ai) & $4.22 \mathrm{a}$ & $1.33 \mathrm{bc}$ & $25.5 \mathrm{ab}$ \\
$\begin{array}{l}\text { Bacillus thuringiensis } \\
\text { (Thuricide HP) }\end{array}$ & $500-1000 \mathrm{~g}$ & $5.44 \mathrm{ab}$ & $2.00 \mathrm{~d}$ & $24.6 \mathrm{ab}$ \\
$\begin{array}{l}\text { Diflubenzuron } \\
\text { (Dimilin WP25) }\end{array}$ & $75-100 \mathrm{~g}$ (ai) & $6.22 \mathrm{ab}$ & $1.66 \mathrm{~cd}$ & $28.3 \mathrm{ab}$ \\
$\begin{array}{l}\text { Chlorofluazuron } \\
\begin{array}{l}\text { Atabron 5EC) } \\
\text { Methamidophos }\end{array}\end{array}$ & $50-100 \mathrm{~g}$ (ai) & $2.89 \mathrm{a}$ & $1.00 \mathrm{ab}$ & $27.3 \mathrm{ab}$ \\
$\begin{array}{l}\text { (Tamaron LC 60) } \\
\text { Azadirachta indica } \\
\text { (Neem oil) }\end{array}$ & $420-500 \mathrm{~g}$ (ai) & $5.55 \mathrm{ab}$ & $2.66 \mathrm{de}$ & $28.1 \mathrm{ab}$ \\
$\begin{array}{l}\text { Hortonia angustifolia } \\
\text { (Root - methanol extract) }\end{array}$ & $11.16 \mathrm{l}$ & $2.11 \mathrm{a}$ & $0.66 \mathrm{a}$ & $18.4 \mathrm{a}$ \\
$\begin{array}{l}\text { Untreated control } \\
\text { CV }\end{array}$ & $2200-4400 \mathrm{~g}$ & $4.78 \mathrm{a}$ & $1.66 \mathrm{~cd}$ & $28.3 \mathrm{ab}$ \\
\hline
\end{tabular}

In each column, the values followed by the same letter do not differ significantly according to DMRT at $\mathrm{p} \leq \_.05$

(ai) $=$ active ingredient 
Immediately before each insecticide application, the number of leaf eating caterpillars in 10 randomly selected and labelled plants in each plot was recorded. Before harvesting, leaf damage was visually estimated using the 1 - 4 scale (Leaf Damage: $0-25 \%=1 ; 26-50 \%=2 ; 51-75 \%=3 ; 76-100 \%=4$ ). After the harvest, the percentage of yield loss due to caterpillar damage was determined.

\section{RESULTS}

\section{Relative toxicity against $A$. craccivora}

Out of the 94 plant extracts (from 55 plants) tested for insecticidal activity against $A$. craccivora, the following showed significant activity $(p \leq 0.05)$ with high percentage mortality (56-90\%) [compared to the Untreated Control (UC)] at $24 \mathrm{HAI}$ (Table 1); the hexane extract of Pleiospermium alatum (St Bk) and the methanol extracts of Costus specious (Rh), Ocimum gratissimum (Rt), Celtis cinnamonea (Lf), Curcuma zedoaria (Tb), and Cestrum aurantiacum (Lf).

A number of reports on the ethnomedical uses and isolation of chemical constituents have been reported for C. specious, C. zedoaria, P. alatum and $Z$. zerumbet. The antifungal principle of the rhizome of $C$. specious has been identified as the methyl ester of $p$-coumaric acid; $;{ }^{7,8}$ this plant which possesses a variety of biological activities, is also a source of diosgenin. ${ }^{9}$ The rhizome of $C$. zedoaria is applied externally on wounds, ulcers, sprains and certain types of dermatitis; C. zedoaria is reported to have antibacterial and antifungal activity. ${ }^{10}$ The anti-fungal agent in $P$. alatum has been identified as seselin; ${ }^{11}$ alkaloids and coumarins have also been isolated from this plant. The powdered rhizome of $Z$. zerumbet is used as an antidiarrhoeic agent; chemical constituents present in this plant are zerumbone, zerumbone oxide, humulene in addition to a number of sesquiterpenes. ${ }^{12,13,14}$

\section{Relative toxicity against $P . x y l o s t e l l a$}

In the laboratory experiments, at $24 \mathrm{HAI}$ significantly high $(\mathrm{p} \leq 0.05)$ insecticidal activity on $P$. xylostella was observed with P. alatum (St Bk) and H. angustifolia (Rt) treatments. At $48 \mathrm{HAI}$, C. aurantiacum (Lf) also showed significant mortality effect on larvae. Significantly low pupation and adult emergence was observed in larvae fed with cabbage leaf disks treated with all the tested plant extracts (Table 2). Second instar larvae fed on leaves treated with C. zedouria ( $\mathrm{Tb}$ ) and C. aurantiacum (Lf) showed some developmental inhibitory activity. These larvae could not develop into the third instar stage and while becoming motionless their anterior part of the body became dark in colour and half-cast exuviae were found at the posterior end of the body. As a result, the second instar did not develop into the third instar and died. 
C. zedoaria (Tb), H. angustifolia (Rt) and C. aurantiacum (Lf) also exhibited significant $(\mathrm{p} \leq 0.05)$ antifeedant activity against $P$. xylostella when compared with the untreated control.

\section{Evaluation of field performance of Hortonia angustifolia methanol extract against cabbage leaf eating caterpillars}

Plots treated with neem oil, chlorofluazuron, triflumuron and the methanol extract of $H$. angustifolia (Rt) reported significantly low ( $\mathrm{p} \leq 0.05)$ number of leaf eating caterpillars when compared with the untreated control and the rest of the treatments with Bacillus thuringiensis and methamidophos (Table 3). All treatments, except methamidophos significantly reduced the leaf damage when compared with the untreated control. However, plots treated with neem oil and chlorofluazuron reported significantly ( $\mathrm{p} \leq 0.05$ ) lower leaf damage than the rest of the treatments. But at harvesting, none of these treatments gave significant reduction $(p \leq 0.05)$ of the yield loss due to caterpillar damage when compared to the untreated control.

\section{DISCUSSION}

From the tested plant extracts hexane extract of $P$. alatum (St Bk), and methanol extract of $C$. specious (Rh), O. gratissimum (Rt), C. cinnamomea (Lf) reported the highest insecticidal activity against the adult Aphis craccivora. The methanol extracts of C. zedoaria (Rt), and $H$. angustifolia (Rt) also showed moderate insecticidal activity against the same insect.

The hexane extract of $P$. alatum (St Bk) and the methanol extract of $H$. angustifolia reported a strong toxic effect on larvae of $P$. xylostella. Methanol extracts of C. zedoaria (Tb), C. aurantiacum (Lf) and H. angustifolia (Rt) showed antifeedant activity and some adverse effect on the development of $P$. xylostella. In a field assay the extract of $H$. angustifolia was significantly more effective on the reduction of cabbage caterpillar compared to an untreated blank whereas it was much less effective in the above reduction compared to some commercially available insecticides.

\section{Acknowledgements}

The authors are grateful to NARESA, Sri Lanka and IFS Sweden for financial support.

\section{References}

1. Mandawa N.B. (1985). Handbook of natural pesticides. CRC Press, Florida, Perkens. 
2. Hill D.S. (1983). Agricultural insect pests of the tropics and their control, 2nd ed. Cambridge, Cambridge University Press.

3. Georghion G.P., Mellon, R.B. (1980). Pesticide resistance in time and space. In: Eds. G.P. Georghion \& T. Saito. Pest resistance of pesticides. Plenum Press, New York, p. 1-46.

4. Hedin P.A. (1983). Plant resistance to insects. ACS Symposium Series 208. American Chemical Society, Washington.

5. Peterson A.(1964). Entomological techniques. In: How to work with insects. Los Angelés, Entomological Reprint Specialists, 435 pp.

6. Bandara K.A.N.P., Peries I.D.R., Kumar V., Karunaratne V. \& Ranasinghe M.A.S.K. (1990). Tropical Agriculture (Trinidad) 67(3): 223-8.

7. Bandara B.M.R., Hewage C.M., Karunaratne V. \& Adikaram N.K.B. (1988). Planta Medica 5: 477-8.

8. Bandara B.M.R., Fernando I.H.S., Hewage C.M., Karunaratne V., Adikaram N.K.B. \& Wijesundara D.S.A. (1989). Journal of the National Science Council of Sri Lanka 17 (1): 1-13.

9. Kaphai B.K., Kapur, S.K., Sarin, Y.K. (1977). Indian Journal of Pharmacology. 39(4): 74-76.

10. Chopra I.C., Jamwal K.S. \& Khajuria B.N. (1954). Indian Journal of Medical Research 42: 381-4.

11. Bandara B.M.R., Gunatilaka A.A.L., Wijeratne E.M.K. \& MacLeod J.K. (1990). Phytochemistry 21: 297-301.

12. Matthes H.W.D., Luu B. \& Ourisson G. (1980). Phytochemistry 19: 264350.

13. Ramaswami S.K. \& Batacharya S.C. (1962). Tetrahedron 18: 575-9.

14. Damodaran N.P. \& Dev. S. (1968). Tetraheotron 24: 4113-22. 